\title{
REFERENCES
}

1. C. Chevalley, Sur la théorie du corps de classes dans les corps finis et corps locaux, Journ. of Coll. of Sciences, Tokyo, II, vol. 9 (1933).

2. - La theorie du corps de classes, Ann. of Math. no. 2 vol. 41 (1940).

3. A. Scholz, Totale Normenreste, die keine Normen sind, als Erzeuger nichtabelscher Körpererweiterungen, no. 2, Crelle J. vol. 182 (1940).

4. A. Weil, Sur la théorie du corps de classes, J. Math. Soc. Japan vol. 3 (1951).

5. G. Whaples, Non-analytic class field theory and Grunwald's theorem, Duke Math. J. vol. 9 (1942).

YAMAGATA, JAPAN

\section{ON SMOOTH LINEAR PARTIAL DIFFERENTIAL EQUATIONS WITHOUT SOLUTIONS ${ }^{1}$}

\section{PHILIP HARTMAN}

1. H. Lewy [4] has recently constructed a system of first order, linear partial differential equations with coefficients of class $C^{\infty}$ having the property that it possesses no "solution" on any domain. By "solution" is meant a solution having uniformly Hölder continuous partial derivatives. Since the "Hölder continuity" requirement on the partial derivatives is rather artificial, there arises the question as to whether or not there is such a system which possesses no $C^{1}$ (or even no weak $\left.L^{2}\right)$ solution on any domain. The object of this note is to answer this question in the affirmative. The desired system will be obtained by a modification of Lewy's example.

Lewy's system involves two (real) unknown functions $\left(u^{1}, u^{2}\right)$ and three (real) independent variables $(s, x, y)$. On putting $u=u^{1}+i u^{2}$, Lewy writes his example in the form

$$
L(u)=F(s, x, y),
$$

where $L$ is the homogeneous, linear, first order, partial differential operator with analytic coefficients,

Received by the editors July 30, 1958.

1 The preparation of this paper was sponsored by the Office of Naval Research. Reproduction in whole or in part is permitted for any purpose of the United States Government. 


$$
L(u)=-\left(u_{x}+i u_{y}\right)+2 i(x+i y) u_{s} .
$$

By a category argument, Lewy shows that for a suitable choice of $F$, say $F=F^{L}(s, x, y)$, of class $C^{\infty}$ for all $(s, x, y)$, (1) does not possess a solution, having uniformly Hölder continuous partial derivatives, on any domain.

2. It will first be shown that

(I) There exist functions $G(s, x, y)$ of class $C^{\infty}$ for all $(s, x, y)$ such that

$$
L(v)=G(s, x, y)
$$

has no $C^{1}$-solution on any domain.

In view of the following lemma, this follows from Lewy's result by choosing $G=\partial F^{L} / \partial s$.

Lemma. Let $F(s, x, y)$ be of class $C^{1}$ on some $(s, x, y)$-domain and let

$$
L(v)=F_{s}(s, x, y)
$$

have a solution $v=v(s, x, y)$ of class $C$. Then (1) possesses a solution $u=u(s, x, y)$ having uniformly Hölder continuous partial derivatives on some domain.

Proof. Let $v=v(s, x, y)$ be a $C^{1}$-solution of (4) on the set $R(r)$ : $\{|s-c| \leqq r\} \times D(r)$, where $D(r):(x-a)^{2}+(y-b)^{2} \leqq r$ and $r>0$. Put

$$
U(s, x, y)=\int_{c}^{s} v(t, x, y) d t,
$$

so that $U_{s}=v$. On integrating the relation (4) with respect to $s$ from $c$ to $s$, it is seen that $U$ satisfies

$$
L(U)=F(s, x, y)-f(x, y),
$$

where $f(x, y)=F(c, x, y)-2 i(x+i y) v(c, x, y)$.

Consider the partial differential equation

$$
-\left(V_{x}+i V_{y}\right)=f(x, y) .
$$

Since $f(x, y)$ is of class $C^{1},(7)$ has a $C^{1}$-solution given by

$$
2 \pi V(x, y)=\iint_{D(r)} f(p, q)(z-w)^{-1} d p d q,
$$

where $w=p+i q$ and $z=x+i y$; Korn, Lichtenstein.

Define the $C^{1}$-function $u=u(s, x, y)$ by

$$
u=U(s, x, y)+V(x, y) \text {. }
$$


Since $V$ does not depend on $s$, the left side of $(7)$ is $L(V)$. Thus the linearity of $L,(6)$ and $(7)$ show that $L(u)=L(U)+L(V)=F$; that is, (9) is a solution of (1).

The partial derivative $u_{s}=U_{s}=v$ is of class $C^{1}$, hence uniformly Hölder continuous.

By (1), $u$ satisfies the partial differential equation

$$
u_{x}+i u_{y}=2 i(x+i y) v(s, x, y)-F(s, x, y),
$$

in which $s$ is considered as a parameter. As the right side of (10) is of class $C^{1}$, standard Korn type of estimates show that $u_{x}$ and $u_{y}$ satisfy a Hölder condition (of any order $<1$ ) with respect to $(x, y)$ which is uniform on $R(d), 0<d<r$; cf., e.g., [1]. By (5) and (9),

$$
u_{p}(s, x, y)=\int_{0}^{s} v_{p}(t, x, y) d t+V_{p}(x, y) \text {, where } p=x \text { or } p=y .
$$

These formulae show that $u_{x}$ and $u_{y}$ satisfy a Lipschitz condition with respect to $s$ which is uniform on $R(r)$. Consequently, $u_{x}$ and $u_{y}$ are uniformly Hölder continuous on $R(d), 0<d<r$. This proves the lemma.

3. Assertion (I) will be improved as follows:

(II) There exist functions $G(s, x, y)$ of class $C^{\infty}$ for all $(s, x, y)$ such that (3) has no strong $L^{2}$-solution on any domain.

It follows from (II) and results of Friedrichs [2] that "strong" can be replaced by "weak" in this assertion.

By a strong $L^{2}$-solution $v=v(s, x, y)$ of (3) on a domain $R$ will be meant a function $v$ of class $L^{2}(R)$ for which there exists a sequence $v^{1}, v^{2}, \cdots$ of functions of class $C^{1}$ satisfying

$$
\left\|v-v^{n}\right\|_{R}+\left\|G-L\left(v^{n}\right)\right\|_{R} \rightarrow 0 \text { as } n \rightarrow \infty,
$$

where $\|\cdots\|_{R}$ is the $L^{2}$-norm on $R$,

$$
\|v\|_{R}^{2}=\iiint_{R}|v|^{2} d s d x d y .
$$

4. By potential theoretical arguments (involving Theorem 2.1, [5, pp. 52-53] and Lemma 2, [3, pp. 393-394]), it can be shown, for example, that $G=\partial^{5} F^{L} / \partial s^{5}$ satisfies the assertion of (II). In fact, if the corresponding equation (3) has a strong $L^{2}$-solution $v=v(s, x, y)$ on some $R=R(r)$, let $v^{1}, v^{2}, \cdots$ be functions of class $C^{1}$ satisfying (11). By the procedure of the proof of the lemma, $v, v^{1}, v^{2}, \cdots$ can be replaced by other functions, say $v, v^{1}, v^{2}, \cdots$, such that $v$ is an 
strong $L^{2}$-solution of (3) with $G=\partial^{4} F^{L} / \partial s^{4}$ on a somewhat smaller $R(r)$, that $v^{n}$ is of class $C^{1}$ and satisfies (11), and that

$$
\iint_{D(r)}\left|v^{n}(s, x, y)\right|^{2} d x d y \leqq M
$$

for all $n$ and $|s-c| \leqq r$. Another repetition of these arguments, changes $G$ to $\partial^{3} F^{L} / \partial s^{3}$ and improves (13) to

$$
\iint_{D(r) \cap C(\epsilon)}\left|v^{n}(s, x, y)\right|^{2} d x d y \leqq M \epsilon^{\lambda}
$$

for some $\lambda(0 / \lambda<1)$, for all $n,|s-c| \leqq r$ and all disks $C(\epsilon)$ of radius $\epsilon$. Another repetition passes to $G=\partial^{2} F^{L} / \partial s^{2}$ and replaces (14) by

$$
\left|v^{n}\right| \leqq M,\left|v^{n}(s, x+h, y+k)-v^{n}(s, x, y)\right| \leqq M\left(|h|^{\lambda}+|k|^{\lambda}\right) .
$$

For $G=\partial F^{L} / \partial s$, one obtains $\left|v^{n}\right| \leqq M$, $v^{n}$ satisfies a Lipschitz condition with respect to $s$ and $v_{x}^{n}, v_{y}^{n}$ satisfy Hölder conditions of order $\lambda$ with respect to $(x, y)$ that are uniform in $n$, and $s, x, y$. Finally, (1) with $F=F^{L}$ has a solution $u=u(s, x, y)$ with uniformly Hölder continuous partial derivatives $u_{s}, u_{x}, u_{y}$.

This procedure also allows the improvement of $L^{2}$ to $L^{p}, p>1$ (but not $p=1$ ) in (II).

5. The details of this procedure will not be given. Instead, it will be indicated how Lewy's arguments can be modified to obtain (II) directly. Let $f(s)$ be a real-valued function of class $C^{1}$ on some interval $|s-c| \leqq r$ and let

$$
L(v)=f_{s}(s)
$$

have a strong $L^{2}$-solution $v=v(s, x, y)$ on

$$
R(r):|s-c| \leqq r, \quad x^{2}+y^{2} \leqq r .
$$

Let $v^{1}, v^{2}, \cdots$ be of class $C^{1}$ on $R(r)$ and satisfy

$$
\left\|v^{n}-v\right\|_{R}+\left\|F^{n}(s, x, y)-f_{s}(s)\right\|_{R} \rightarrow 0 \text { as } n \rightarrow \infty,
$$

where $L\left(v^{n}\right)=F^{n}$. Then, as in [4, pp. 155-156], for $t>0$,

$$
U^{n}(s, t)=i \int_{0}^{2 \pi}(x+i y) v^{n}(s, x, y) d \theta, \text { where } x+i y=t^{1 / 2} e^{i \theta},
$$

satisfies

$$
U_{s}^{n}+i U_{t}^{n}=\frac{1}{2} \int_{0}^{2 \pi} F^{n}(s, x, y) d \theta, \text { where } x+i y=t^{1 / 2} e^{i \theta} .
$$

From Schwarz's inequality, 


$$
\left|U_{s}^{n}+i U_{t}^{n}-\pi f_{s}(s)\right|^{2} \leqq \frac{1}{2} \pi \int_{0}^{2 \pi}\left|F^{n}-f_{s}\right|^{2} d \theta,
$$

and from $d \theta d t d s=2 d x d y d s$, it is easy to show that $U^{1}(s, t), U^{2}(s, t), \cdots$ has a (strong) $L^{2}$-limit $U(s, t)$ on $|s-c|<r, 0<t<r$ and that

$$
V(s, t)=U(s, t)-\pi f(s)
$$

is a strong $L^{2}$-solution of $V_{s}+i V_{t}=0$. Hence, $V(s, t)$ is an analytic function of $s+i t$.

By (19) and Schwarz's inequality,

$$
\left|U^{n}(s, t)\right|^{2} \leqq 2 \pi t \int_{0}^{2 \pi}\left|v^{n}\right|^{2} d \theta
$$

so that $|U(s, t)|^{2} / t$ is Lebesgue summable on $|s-c|<r, 0<t<r$. Hence, as $t \rightarrow+0$ through some sequence $t_{1}, t_{2}, \cdots$, one has $\int|U(s, t)|^{2} d s \rightarrow 0$ or, equivalently,

$$
\int_{c-r}^{c+r}|V(s, t)+\pi f(s)|^{2} d s \rightarrow 0 \text { as } t=t_{n} \rightarrow 0 .
$$

In other words, the boundary values (in the sense of (22)) of the analytic function $V(s, t)$, for $t=0$, are given by the real-valued continuous function $-\pi f(s)$.

Standard proofs (cf., [7, pp. 155-156]) of the Schwarz reflection principle show that $V(s, t)$ can be continued by reflection so as to be analytic for $|s-c|<r,|t|<r$. In particular, (16) has no strong $L^{2}$ solution on (17) unless the (real-valued) $f(s)=-V(s, 0) / \pi$ is an analytic function of $s$ for $|s-c|<r$. This result corresponds to Lewy's theorem, $[4$, p. 155].

(It is not clear that the argument leading to this assertion permits the relaxation of $L^{2}$ to $L^{p}, p>1$, even though the corresponding assertion follows from the procedure described in $\$ 4$.)

6. The proof of (II) can now be obtained by Lewy's construction and arguments in [4, pp. 157-158]. It is only necessary to replace his class $H_{n m}^{1}$ by a class $h_{m}$ of functions $u$ subject to $\|u\|_{R} \leqq m$. For if $u^{1}, u^{2}, \cdots$ are functions of class $L^{2}$ on a domain $R$ such that $\left\|u^{n}\right\|_{R}$ $\leqq m, u=u^{n}$ is a strong $L^{2}$-solution of $L\left(u^{n}\right)=F^{n}(s, x, y)$ on $R$ and there exists an $F(s, x, y)$ on $R$ satisfying

$$
\left\|F^{n}-F\right\|_{R} \rightarrow 0 \text { as } n \rightarrow \infty,
$$

then (1) has a strong $L^{2}$-solution on $R$. In fact, it can be supposed that $u^{1}, u^{2}, \cdots$ has a weak limit $u$ in $L^{2}(R)$ and $\|u\|_{R} \leqq m$. Then, by a 
theorem of Banach-Saks, $u^{1}, u^{2}, \cdots$ can be replaced by a subsequence, say $u^{1}, u^{2}, \cdots$, such that $v^{n}=\left(u^{1}+\cdots+u^{n}\right) / n$ tends strongly to $u$ in $L^{2}(R)$; cf. [6, pp. 80-81]. Since $v^{n}$ is a strong $L^{2}-$ solution of $L\left(v^{n}\right)=\left(F^{1}+\cdots+F^{n}\right) / n$, it follows that $L\left(v^{n}\right)$ tends strongly to $F$. Hence, $u$ is a strong $L^{2}$-solution of (1) on $R$.

7. REMARK. If $f(s)$ is a real-valued function of class $C^{1}$ for $s$ near $c$, then

$$
P(v)=f_{8}(s)(x-i y),
$$

where $P(v)=-\left(v_{x}+i v_{y}\right)+4 i(x-i y)(x+i y)^{2} v_{s}$, has no strong $L^{1}$ solution in any neighborhood of $(s, x, y)=(c, 0,0)$ unless $f(s)$ is analytic at $s=c$. This follows from the argument of $\$ 5$ after multiplying the equation $P\left(v^{n}\right)=F^{n}(s, x, y)$ by $e^{i \theta}$, integrating with respect to $\theta$, and letting

$$
U^{n}(s, t)=4 i \int_{0}^{2 \pi}(x+i y)^{2} v^{n} d \theta, \text { where } \quad x+i y=t^{1 / 4} e^{i \theta}
$$

and $t>0$, to obtain the equation

$$
t^{1 / 4}\left(U_{s}^{n}+i U_{t}^{n}\right)=\int_{0}^{2 \pi} e^{i \theta} F^{n}(s, x, y) d \theta, \text { where } x+i y=t^{1 / 4} e^{i \theta} .
$$

\section{REFERENCES}

1. S. S. Chern, An elementary proof of the existence of isothermal parameters on a surface, Proc. Amer. Math. Soc. vol. 6 (1955) pp. 771-782.

2. K. O. Friedrichs, The identity of weak and strong extensions of differential operators, Trans. Amer. Math. Soc. vol. 55 (1944) pp. 132-151.

3. P. Hartman, On integrating factors and on conformal mappings, ibid. vol. 87 (1958) pp. 387-406.

4. H. Lewy, An example of a smooth linear partial differential equation without solution, Ann. of Math. vol. 66 (1957) pp. 155-158.

5. C. B. Morrey, Multiple integral problems in the calculus of variations, California, 1943.

6. F. Riesz and B. Sz.-Nagy, Leçons d'analyse fonctionnelle, Budapest, 1952.

7. E. C. Titchmarsh, The theory of functions, Oxford, 1939.

University of California, Los ANGeles and

The Johns Hopkins UnIVERSITY 